\title{
Nitrogen Deficiency in Culture Affects the Metabolic Pathways in Amphora Coffeaeformis for Biodiesel Production
}

\author{
Kang Wang \\ Yantai Institute of Coastal Zone Research \\ Yulin Cui \\ Yantai Institute of Coastal Zone Research \\ Chunxiao Meng ( $\sim$ mengchunxiao@126.com ) \\ Binzhou Medical University \\ Zhengquan Gao \\ Binzhou Medical University \\ Song Qin \\ Yantai Institute of Coastal Zone Research
}

\section{Research}

Keywords: Amphora coffeaeformis, nitrogen deprivation, lipid accumulation, RNA-seq, TAG

Posted Date: August 27th, 2021

DOI: https://doi.org/10.21203/rs.3.rs-814667/v1

License: (a) (i) This work is licensed under a Creative Commons Attribution 4.0 International License. Read Full License 


\section{Abstract}

\section{Background}

Amphora coffeaeformis, a unicellular diatom, can accumulate large amounts of lipids under nitrogen $(\mathrm{N})$ limitation, because of which it can act as a promising raw material for biodiesel production. However, the molecular mechanism underlying lipid accumulation in $A$. coffeaeformis remains unknown.

Results

In this study, we investigated the mechanism underlying lipid accumulation under $\mathrm{N}$ deprivation conditions in $A$. coffeaeformis using RNA-seq. The results showed that the total lipid (TL) content of $A$. coffeaeformis in normal f/2 medium was $28.22 \%$ (TL/DW), which increased to $44.05 \%$ after 5 days of $\mathrm{N}$ deprivation, while the neutral lipid triacylglycerol (TAG) content increased from $10.41 \%$ (TAG/DW) to $25.21 \%$. The transcriptional profile showed that 591 genes were up-regulated, with false discovery rate cutoff of $0.1 \%$, and 1,021 genes were down-regulated, indicating that $\mathrm{N}$ deprivation induced wide-ranging reprogramming of regulation, and that most physiological activities were repressed. In addition, ribosome biogenesis, carbon fixation, and photosynthesis in A. coffeaeformis were considerably affected by $\mathrm{N}$ deprivation.

\section{Conclusions}

In summary, the findings shed light on the molecular mechanisms of neutral lipid accumulation and revealed the key genes involved in lipid metabolism in $A$. coffeaeformis, which will be useful in designing strategies for improving microalgal biodiesel production.

\section{Background}

With intensification of the world energy crisis and increasing interest in reducing carbon dioxide emissions, the interest in biofuels has received impetus [1]. Microalgae have become one of the alternative raw materials for biofuel production due to their high carbohydrate and fat content, fast growth rate, and strong resistance to the environment [2,3]. Reports have shown that the photosynthetic productivity of microalgae is several folds higher than that of higher plants, which also increases the fatty acid synthesis in certain microalgae $[4,5]$. Furthermore, microalgae can also resolve the problem of land competition between traditional oil crops and food crops. The increase in demand for energy and the continuous progress in new biofuel production routes have increased research on the potential of microalgae as a third-generation biofuel [1].

Generally, oil levels $20-50 \%$ of dry mass are common in microalgae, which can reach up to $70 \%$ of dry mass when cells are subject to physiological stress conditions or unfavorable environment, such as nutrient limitation or photo-oxidative stress [5]. Nutrient availability is of considerable importance for the growth and primary production of microalgae [6]. Typical nutrient limitation in nature affects the supply 
of nitrogen, phosphate, and/or silicate [7]. Nitrogen is the main nutrient element that affects the growth and oil content of microalgae [8]. However, although microalgae can attain the maximum total biomass under high nitrogen concentrations, the total lipid content decreases [9]. In contrast, nitrogen limitation can lead to decrease in protein content and a relative increase in carbohydrate and/or lipid storage, and can also result in reduction in growth rate and photosynthetic efficiency, thereby affecting the final biomass of microalgae $[10,11]$. In addition, nitrogen is also necessary for some microalgae to synthesize unsaturated fatty acids. Insufficient nitrogen not only increases the content of saturated fatty acids in the algae cells, but also reduces the content of PUFAs [11]. Yang et al. [12] found that the content of polyunsaturated fatty acids in Phaeodactylum tricornutum decreased when the nitrogen source was insufficient. The main reason may be that the lack of nitrogen reduces the synthesis of amino acids, which in turn reduces the production of pigment bodies (rich in protein), which leads to reduction in the demand for phospholipids and glycerol lipids by algal cells.

Diatoms account for $40 \%$ of the primary productivity of marine ecosystems, and some species, such as $P$. tricornutum, Amphora sp., and Nitzschia sp., are known to accumulate neutral lipids [12]. Furthermore, diatoms are metabolically versatile, as they can synthesize and accumulate wide ranges of valuable compounds, such as PUFAs $[13,14]$. Therefore, they have attracted both biological and medical attention. Recently, the marine diatom Amphora coffeaeformis has emerged as a potential microalgal energy source. It grows rapidly, has a short life cycle, and accumulates TAGs in the late exponential phase [15]. Although previous studies have successfully demonstrated the roles of $\mathrm{N}$ limitation in lipid accumulation in A. coffeaeformis [15], the underlying metabolic pathways remain largely unknown. Comprehensive analyses of gene expression using RNA-sequence technology will assist in understanding the metabolic pathways associated with cellular responses to $\mathrm{N}$ limitation.

In this study, the transcriptome of $\mathrm{N}$-deprived $A$. coffeaeformis was investigated to examine differential regulation of genes associated with pathways of interest. In-depth comparison of the RNA-seq data revealed significant differences in gene expression associated with lipid biosynthesis. This is the first report to examine differential gene expression in response to $\mathrm{N}$ deprivation in $A$. coffeaeformis, which will expand understanding regarding microalgal lipid metabolism and biosynthesis.

\section{Results And Discussion}

\subsection{N deprivation restrained the growth and physiological status}

To monitor growth, single cells of $A$. coffeaeformis were separated in three petri dishes and observed using an inverted microscope every $24 \mathrm{~h}$. The growth curve was shown in Fig. 1. The growth rate was 0.22 per day, the division was 0.32 per day, and the resulted doubling time was 3.15 days. The growth rate of A. coffeaeformis is comparable with those of the other known Amphora algae [16], which indicated that the condition in this experiment was suitable for this species. 
As previously reported in other oleaginous microalgae [17], the lack of nitrogen inhibits the growth and significantly shortens the time required for $A$. coffeaeformis in the stable phase to enter the recession stage. The growth rate of $A$. coffeaeformis in $\mathrm{f} / 2-\mathrm{N}$ medium was 0.06 per day and the doubling time extended to 11.67 days (Fig. 1). For $A$. coffeaeformis culture in the stable phase, $\mathrm{N}$ deprivation restrained growth and attachment status. In Fig. 2, the $A$. coffeaeformis in normal f/2 medium was uniformly attached to the bottom of Petri dishes; in contrast, some cells fell off the substratum after 5 days of $\mathrm{N}$ deprivation. The stable phase of $A$. coffeaeformis under normal condition was maintained for more than 20 days, following which, some cell clusters fell off, were suspended in the medium, and died (recession stage). However, $\mathrm{N}$ deprivation shortened the stable phase of $A$. coffeaeformis to 7-8 days and the recession stage to 10 days.

The response of the photosynthetic performance to growth in nitrogen-rich and nitrogen-free media is particularly important for improving our understanding regarding the metabolic and physiological differences in diatoms under $\mathrm{N}$ deprivation [11]. The photosynthesis performance parameters can directly reflect the growth status of microalgae, providing detailed information regarding the cellular physiology of the algae under $\mathrm{N}$ deprivation [18]. Hence, the photosynthetic activity was examined during $\mathrm{N}$ deprivation to monitor the physiological status of $A$. coffeaeformis. The maximum PSIl photochemical efficiency Fv/Fm and Etr characterize the physiological response of microalgal cells to changes in environmental conditions, such as nutrient starvation or photoinhibition [11]. In this study, Fv/Fm and Etr declined in $\mathrm{N}$ deprived medium (Fig. 3). Especially the character YII of A. coffeaeformis after 6 days' $\mathrm{N}$ deprivation declined dramatically (0.46 to 0.19 ). Obviously, the physiological activity of $A$. coffeaeformis under $\mathrm{N}$ deprivation was inhibited owing to inhibition of photosynthetic activity.

\section{$2.2 \mathrm{~N}$ deprivation promoted lipid accumulation in $A$. coffeaeformis}

The accumulation of lipids in microalgae under $\mathrm{N}$ deficiency has studied for decades. Studies have shown that the growth of microalgae (i.e., synthesis of protein and nucleic acids) is limited under $\mathrm{N}$ deficiency, although the process of carbon assimilation is always ongoing, which channelizes more carbon into lipid metabolism $[10,11]$. As an important component of lipids, TAGs do not contain nitrogen. Therefore, despite the lack of nitrogen in microalgae under conditions supporting ongoing carbon assimilation, TAGs can accumulate rapidly in cells. Converti et al. [19] showed that when the nitrogen source in the culture medium was reduced by $75 \%$, the total lipid content in Nannochloropsis oculata increased from $7.9-15.31 \%$, while the total lipid content of Chlorella vulgaris increased from $5.9-16.41 \%$. In the present study, the total lipid content of $A$. coffeaeformis in normal $\mathrm{f} / 2$ medium was $28.22 \%$ (TL/DW), which increased to $44.05 \%$ after 5 days of $\mathrm{N}$ deprivation, while the neutral lipid TAG content increased from $10.41 \%$ (TAG/DW) to $25.21 \%$ (TAG/DW) (Fig. 4). The lipid content in A. coffeaeformis was as high as that reported in other Amphora species.

Distinct difference between the fatty acid compositions of $A$. coffeaeformis in $\mathrm{f} / 2$ medium and under $\mathrm{N}$ deprivation was not observed (Table 1). Surprisingly, the fatty acid compositions of TAG in N-deprived $A$. 
coffeaeformis differed considerably from that of the control (Table 2). The saturated fatty acid contents increased dramatically from 57.94-81.21\%, especially that of the long chain fatty acid C16:0, C18:0, and C20:0. Furthermore, the content of the unsaturated fatty acids decreased. These results indicated that the key enzymes in the TAG synthesis pathway may facilitate metabolism of saturated fatty acids and as a result, the unsaturated fatty acids flowed to the other lipids, with the exception of TAG. 
Table 1

Fatty acid composition of $A$. coffeaeformis following $\mathrm{N}$ deprivation.

\begin{tabular}{|lll|}
\hline Fatty acids & control & $-\mathbf{N}(\mathbf{5} \mathbf{d})$ \\
\hline $12: 00$ & 0.17 & 0.165 \\
\hline $13: 00$ & 0.1 & 0.09 \\
\hline $14: 00$ & 10.02 & 10.04 \\
\hline $14: 01$ & 0.15 & 0.13 \\
\hline $14: 01 \mathrm{t}$ & 2.17 & 2.13 \\
\hline $14: 01 \mathrm{t}$ & 4.57 & 4.31 \\
\hline $15: 00$ & 2.81 & 2.66 \\
\hline $16: 00$ & 23.48 & 23.41 \\
\hline $16: 01$ & 24.28 & 25.31 \\
\hline $16: 01 \mathrm{t}$ & 0.62 & 0.61 \\
\hline $16: 02$ & 1.24 & 1.32 \\
\hline $16: 03$ & 2.27 & 2.32 \\
\hline $18: 00$ & 1.57 & 1.245 \\
\hline $18: 01$ & 2.34 & 2.32 \\
\hline $18: 01 \mathrm{t}$ & 1.3 & 1.175 \\
\hline $18: 02$ & 2.76 & 2.715 \\
\hline $18: 03$ & 0.06 & 0.06 \\
\hline $19: 01$ & 0.76 & 0.79 \\
\hline $18: 03$ & 0.15 & 0.15 \\
\hline $20: 00$ & 0.07 & 0.07 \\
\hline $20: 03$ & 0.24 & 0.25 \\
\hline $20: 04$ & 8.06 & 7.95 \\
\hline $20: 05$ & 8.67 & 8.775 \\
\hline $24: 00$ & 0.97 & 0.96 \\
\hline $24: 01$ & 0.56 & 0.06 \\
\hline
\end{tabular}


Table 2

Fatty acid composition in TAGs with significant changes following $\mathrm{N}$ deprivation.

\begin{tabular}{|lll|}
\hline Fatty acids & Normal & $\mathbf{- N}(\mathbf{5} \mathbf{~})$ \\
\hline $14: 0$ & 7.52 & 4.99 \\
\hline $16: 0$ & 30.72 & 33.08 \\
\hline $16: 1$ & 23.17 & 7.3 \\
\hline $16: 1 \mathrm{t}$ & 2.77 & 5.51 \\
\hline $18: 0$ & 10.29 & 29.14 \\
\hline $18: 1$ & 3.92 & 6.81 \\
\hline $18: 1 \mathrm{t}$ & 1.97 & 1.47 \\
\hline $18: 2$ & 1.82 & 5 \\
\hline $18: 3$ & 0.34 & 4.56 \\
\hline $20: 0$ & 1.06 & 3.66 \\
\hline
\end{tabular}

\subsection{High-throughput analysis of $A$. coffeaeformis under $\mathrm{N}$ deprivation}

To obtain in-depth knowledge regarding the mechanisms, comprehensive analyses such as analysis of gene expression should be used. Therefore, RNA-seq was used to assess differences in gene expression between the $\mathrm{N}$-deprived $A$. coffeaeformis and the control. In this regard, we hypothesized that the molecular mechanism underlying the effect of $\mathrm{N}$ deprivation on lipid accumulation in $A$. coffeaeformis could be determined by studying the changes in gene expression. Owing to the lack of reported genome sequences, the RNA-seq analysis was performed using the $P$. tricornutum genome as the reference. Approximately 55 million transcripts were sequenced in each sample, and more than $80 \%$ transcripts mapped to the $P$. tricornutum genome, and the high similarity to the transcripts of other microalgae species indicated the validity of the data.

In this study, the comprehensive transcriptome data of $A$. coffeaeformis under $\mathrm{N}$ deprivation was compared with that under normal conditions, and the complete data is available at http://www.ncbi.nlm.nih.gov/bioproject/753251. To identify functional categories of unigenes, KEGG analysis was performed in accordance of all 110,454 unigenes of $A$. coffeaeformis. And the analyses suggested that the putative functional roles of each transcript obtained were involved in diverse cellular functions, i.e., general metabolism, organismal systems, genetic information processing, environmental information processing, and cellular processes (Fig. 5). The category of amino acid metabolism accounted for 2,877 unigenes, whereas lipid metabolisms contained 1,273 transcripts. Moreover, 
transcripts associated with genetic information processing also constituted a large fraction in the unigenes, which demonstrated the importance of the transcriptional regulation under $\mathrm{N}$ deprivation.

\subsection{DEGs in response to $\mathrm{N}$-deprived conditions}

To evaluate the effect of $\mathrm{N}$-deprived condition on A. coffeaeformis transcripts, DEGs that showed over two-fold changes in their expression compared with the control were selected for further analysis, are shown in the volcano plot (Additional file 1: Fig S1). In total, 591 genes were found to be up-regulated, with a false discovery rate (FDR) cutoff of $0.1 \%$, and 1,021 genes were down-regulated, indicating that $\mathrm{N}$ deprivation induced wide re-programming of regulation, and in particular, many genes were widely downregulated, which may illustrate that most physiological activities were repressed under the conditions of the lack of nitrogen in the environment.

KEGG pathway enrichment analysis of the DEGs was performed for demonstrating the putative functional roles of the identified DEGs in further detail. KEGG analysis for DEGs classified them as being associated with different functional categories, in which multiple genes were significantly up-regulated or down-regulated, suggesting possible mechanisms involved in the response to $\mathrm{N}$ deprivation. For example, genes mediating metabolism of nitrogenous compounds and ribosome biogenesis were up-regulated, while genes encoding enzymes required for photosynthesis, biosynthesis of unsaturated fatty acids, carbon fixation, glycolysis and gluconeogenesis, porphyrin and chlorophyll metabolism were generally down-regulated under $\mathrm{N}$ deprivation (Fig. 6). GO analysis suggested similar patterns to the KEGG analysis. Hence, it can be speculated that certain biochemical processes might be involved in adaptation to the lack of nitrogen.

Genes with $\log _{2}$-fold differences in RNA expression between the control and N-deprived A. coffeaeformis were considered Global changes in major categories of genes involved in various pathways, reflecting general transcriptional responses to $\mathrm{N}$ deprivation, are shown in Fig. 7. And the fold changes in the expression of some genes encoding enzymes involved in $A$. coffeaeformis metabolisms following $\mathrm{N}$ deprivation were summarized in Additional file 2 (Table S1).

\subsection{Transcripts involved in nitrogen assimilation increased}

Nitrogen element is basic component constituting nucleic acids, proteins, and other nitrogen-containing compounds, the primary effect of $\mathrm{N}$ deprivation is the reduced availability of nitrogen and eventually cause the synthesis of nitrogenous compounds hindered. Unsurprisingly, therefore, the lack of nitrogen strongly affected transcript levels of genes involved in nitrogen assimilation and metabolism (Additional file 2: Table S1a). For example, the transcripts encoding three nitrate reductase (NADH-nitrate reductase, EC 1.7.1.1; assimilatory NAD (P)H-nitrate reductase, EC 1.7.1.2; assimilatory NADPH-nitrate reductase, EC 1.7.1.3) that catalyzes the reaction of nitrate to nitrite were increased with 3.4-fold changes. There was a 2.1-fold increase in transcripts of glutamine synthase (EC 6.3.1.2), a key enzyme that plays an important role in the efficient use of nitrogen sources and nitrogen metabolism. Meanwhile, significant increases 
were also observed in transcripts encoding three of at least six ammonium transporters present in the diatom, which can transport ammonium ions across the cell membrane and are known to be activated by $\mathrm{N}$ deprivation [20]. In addition, some critical nitrogen metabolism enzymes, such as NADH-glutamate synthase (EC 1.4.1.14) and ferredoxin-glutamate synthase (EC 1.4.7.1) also showed significant increases (1.5-fold and 1.6-fold, respectively) in transcript abundance. Hence, it can be speculated that $\mathrm{N}$ deprivation induces homeostatic responses, including the activation of glutamine synthesis pathway and the increases in the ability of cells to utilize trace amounts of nitrogen resources, and also the possible redistribution of intracellular nitrogen such as increased amino acid catabolism [21]. In summary, these results indicated that $\mathrm{N}$ deprivation caused many changes in metabolic pathways, especially in amino acid and nitrogen metabolism, which may be a stress response requiring significant changes in gene expression.

\subsection{Transcripts of photosynthesis-related genes decreased}

As mentioned earlier, decreased availability of nitrogen element lowered growth rates, photosynthetic efficiency and had consequences on cell production. In particular, for photosynthetic cells, inhibition or stop of photosynthesis means stagnation of cell growth or death. In fact, the transcripts encoding proteins related to photosynthesis were investigated under nitrogen deficiency, and the levels of most transcripts were decreased (Additional file 2: Table S1b), which implied the stagnation of photosynthesis. Notably, transcript levels of ferredoxin-NADP ${ }^{+}$reductase (EC 1.18.1.2) that catalyzes the last electron transfer (from photosystem I to NADPH) during photosynthesis also decreased (4.8-fold). In accordance with theoretical expectations, the down-regulation of transcripts of light harvesting complexes may reduce photosynthetic rates of the cells under the conditions that do not consider changes in other levels [22].

The decreased transcripts of genes encoding photosynthetic proteins was consistent with expectations, as many previous reports also showed these results [23,24], which indicates the assimilation of nitrogen has profound effects on photosynthetic metabolism [25]. Meanwhile, the decreases of physiological parameters associated with photosynthesis, such as Fv/Fm and Etr (Fig. 3), are also consistent with the down-regulation of transcripts of light harvesting complexes. These physiological parameters significantly decreased, indicating that the decrease of photosynthetic protein under nitrogen deprivation may impair the PSII reaction centers, and eventually resulted in the decrease of both chlorophyll fluorescence yield and photochemical activity of PSII reaction centers [26].

\subsection{Carbon assimilation and carbon fluxes towards TAG accumulation increased}

There are three carbon fixation pathways for photosynthetic organisms: 1) Calvin-Benson-Bassham (CBB) cycle, 2) $\mathrm{C}_{4}$-dicarboxylic acid cycle, and 3) Crassulacean acid metabolism [27, 28]. To our best of knowledge, so far, only the CBB cycle of microalgae has been reported [29]. However, it was found that two genes encoding phosphoenol-pyruvate carboxylase (PEPC, EC 4.1.1.31) were up-regulated (1.53-fold and 1.46-fold, respectively) in the genome of $A$. coffeaeformis under $\mathrm{N}$ deprivation, which catalyzed the 
synthesis of OAA from phosphoenolpyruvate (PEP) (Additional file 2: Table S1c). In fact, PEPC is usually involved in carbon fixation as a key enzyme in $\mathrm{C}_{4}$-dicarboxylic acid cycle [22]. Hence, it can be speculated that the $\mathrm{C}_{4}$ photosynthetic pathway exist in $A$. coffeaeformis. PEPC catalyzes an irreversible carboxylation in the presence of $\mathrm{HCO}_{3}{ }^{-}$and $\mathrm{Me}^{2+}$, the increase of the transcripts of these two PEPCs observed in this study may suggest that it can take up inorganic carbon and possesses a $\mathrm{CO}_{2}$ concentration mechanism [30].

Pyruvate-phosphate dikinase (PPDK, EC 2.7.9.1) is also a key enzyme in $\mathrm{C}_{4}$-dicarboxylic acid cycle, which catalyzes the synthesis of PEP from pyruvate, and its expression significantly affects the accumulation of photosynthetic products in $\mathrm{C}_{4}$ plants [31]. The transcripts of two PPDK genes in A. coffeaeformis under $\mathrm{N}$ deprivation were significantly decreased (1.7-fold and 2.2-fold, respectively), implicating that the decrease of photosynthetic rate. Hence, it can be predicted that the reduction of PPDK level reduces the consumption of pyruvate, thereby enables more pyruvate to synthesize acyl-CoA, the precursor of fatty acid, while the excessively expressed PEPCs can use the available PEP for carbon fixation. Moreover, PPDK is also involved in PPDK-mediated gluconeogenesis [32], which indicates that the decreases of PPDKs expression may inhibit the activation of gluconeogenesis.

Notably, the significant increased transcripts of a gene encoding NADP ${ }^{+}$-malic enzyme (ME, EC 1.1.1.40) was also observed. It is speculated that this gene may be involved in the carbon fixation pathway in $A$. coffeaeformis, and is predicted to be localized in the chloroplast. ME catalyzes the irreversible decarboxylation of malate to pyruvate in photosynthetic cells with the formation of NADPH from NADP', which is the rate-limiting step of fatty acid biosynthesis. In fact, many previous reports have confirmed the promoting effect of ME overexpression on fatty acid synthesis. For example, the overexpression of endogenous NADP-dependent ME in N. salina enhanced the lipid production, and the report also analyzed the total carbon concentration and NADPH/NADP ${ }^{+}$ratio, which were found to be enhanced in the transformants [33]. Hence, the up-regulation of ME in A. coffeaeformis under $\mathrm{N}$ deprivation may increase the NADPH production, thereby providing both reducing power and cofactors for reactions catalyzed by enzymes involved in fatty acid synthesis such as ACCase, fatty acid synthase, and eventually leading to increases in TAG accumulation. Similarly, overexpression of ME gene from $P$. tricornutum in $C$. pyrenoidosa reportedly showed higher ME enzymatic activity, which subsequently promoted fatty acid synthesis, and the neutral lipid content was significantly increased by up to 3.2-fold [34]. Taken together, the dramatic increase in ME transcription observed in this study under $\mathrm{N}$ deprivation could contribute substantially to the accumulation of neutral lipids in A. coffeaeformis.

Fructose-1,6-bisphosphatase (FBP, EC 3.1.3.11) catalyzes the conversion of fructose-1,6-biphosphate to fructose-6-phosphate, which is a key enzyme in CBB cycle and gluconeogenesis. A. coffeaeformis appears to possess at least three isoforms of FBPs, and transcript levels of genes encoding these three isoforms declined under $\mathrm{N}$ deprivation, 3.4-, 1.3- and 2-fold, respectively, indicating that CBB cycle and gluconeogenesis were markedly inhibited and carbon flux was re-directed towards TAG accumulation under $\mathrm{N}$ deprivation. Notably, the transcript abundance of pyruvate carboxylase (PC, EC 6.4.1.1), a 
tricarboxylic acid (TCA) cycle enzyme identified in the $A$. coffeaeformis genome, decreased 3-fold. In fact, the PC catalyzed reaction is the main supplementary reaction for the supply of OAA in the TCA cycle, and the reaction is activated when acetyl-CoA is abundant. Therefore, the down-regulation of PC transcript level may indicate the decrease of acetyl CoA flowing into TCA cycle, while a large amount of acetyl-CoA was used to synthesize TAG. In addition, the significantly differences of transcripts encoding two potentially rate-limiting enzymes of the TCA cycle, citrate synthase (EC 2.3.3.1) and isocitrate dehydrogenase (EC 1.1.1.42), were not observed. However, the transcript levels of genes encoding two succinate dehydrogenases (SDH, EC 1.3.5.1), another key enzyme in TCA cycle, were significantly decreased (2.2-fold and 1.1-fold, respectively), implying that the metabolic flux through the TCA cycle could be reduced under $\mathrm{N}$ deprivation. In summary, the transcriptional changes in carbon assimilation of A. coffeaeformis under $\mathrm{N}$ deprivation may substantially increase carbon influxes, thereby providing a rich source of substrate for TAG production.

\subsection{Increased GPAT levels promoted TAG biosynthesis}

In general, oil-bearing microalgae significantly accumulated neutral lipids after nitrogen induction. Hence, to further determine the precise mechanism via which the microalga up-regulated gene expression under $\mathrm{N}$ deprivation, genes involved in TAG and fatty acid biosynthesis were studied. Notably, $\mathrm{N}$ deprivation increased the transcript levels of genes associated with TAG biosynthesis. In particular, the expression of glycerol-3-phosphate 0-acyltransferase (GPAT, EC 2.3.1.15) that catalyze the first committed step of TAG biosynthesis increased (1.3-fold); thus, the increase in their mRNA abundance under $\mathrm{N}$ deprivation may have increased TAG levels.

Notably, transcripts of gene encoding lysophosphatidic acid-acyltransferase (LPAAT, EC 2.3.1.51) that catalyzes the second step of TAG biosynthesis decreased (1.2-fold). Interestingly, the significantly differences of transcripts encoding diacylglycerol acyltransferase (DGAT, EC 2.3.1.20) that catalyzes the final committed step of TAG biosynthesis, were not observed; Meanwhile, the mRNA levels of another enzyme, responsible for the last step of TAG biosynthesis, phospholipid: diacylglycerol acyltransferase (PDAT, EC 2.3.1.158), decreased 1.7-fold. These findings suggest that the overexpression of GPAT seems

to be a key factor in TAG biosynthesis of $A$. coffeaeformis. In fact, some previous reports also showed the same phenomenon. For example, only transcripts of GPAT1 and GPAT2 were increased among fatty acid and TAG biosynthesis genes under TAG accumulation conditions in Cyanidioschyzon merolae [35]. Similarly, overexpression of GPAT1 and GPAT2 in C. merolae resulted in up to a 56.1 -fold increases in seed oil content [36]. Taken together, the reaction catalyzed by the ER-localized GPAT is a rate-limiting step for TAG synthesis in A. coffeaeformis, and would be a potential target for improvement of TAG productivity in microalgae.

\section{Conclusion}

Microalgae accumulate TAG, a promising feedstock for biodiesel production, under unfavorable environmental or stress conditions for their growth. In the present study, the mechanism underlying lipid accumulation under $\mathrm{N}$ deprivation conditions in $\mathrm{A}$. coffeaeformis was investigated. $\mathrm{N}$-deprived cultures of 
A. coffeaeformis showed up to 2.4-fold increases in neutral lipid contents per cell, slightly higher total lipid content than controls. Moreover, the lipid composition of the cultures under $\mathrm{N}$ deprivation was more suitable for biodiesel production as the higher proportions of saturated fatty acids. To further determine the changes of gene expression under $\mathrm{N}$ deprivation, the comparative transcriptome analysis for $A$. coffeaeformis in response to $\mathrm{N}$ deprivation was performed. We identified 1,612 DEGs, and further in silico functional analyses indicated that multiple genes in different functional categories were differentially expressed under $\mathrm{N}$ deprivation. KEGG pathway analysis and Go analysis were conducted for the DEGs, and found that energy resources necessary for carbon fixation and energy demand associated with the TAG synthesis could be replenished from other sources such as reduction of gluconeogenesis and TCA cycle. In addition, we also find that GPAT may be a rate-limiting step in TAG biosynthesis pathway of $A$. coffeaeformis, which would be a potential target for improvement of TAG productivity in microalgae. Hence, these findings provide indications of mechanisms that contribute to the high lipid contents of microalgae under $\mathrm{N}$ deprivation.

In summary, results of this study show that $A$. coffeaeformis cultures with high levels of neutral lipids and increased cell proliferation rates can be obtained by $\mathrm{N}$ deprivation, and provide a molecular basis for the mechanisms underlying the responses to $\mathrm{N}$ deprivation in $A$. coffeaeformis, which will help to formulate appropriate optimization strategies in oil-bearing microalgae to improve the production of neutral lipids.

\section{Materials And Methods}

\subsection{Microalga strain and growth condition}

The $A$. coffeaeformis strain was collected from the Atlantic coastal zone of Namibia. The purified $A$. coffeaeformis was incubated in petri dishes containing $20 \mathrm{~mL} \mathrm{f} / 2$ medium. The culture condition was as follows: $30^{\circ} \mathrm{C}, 100-110 \mu \mathrm{mol}$ photons $\mathrm{m}^{-2} \mathrm{~s}^{-1}, 12 \mathrm{~h} / 12 \mathrm{~h}$ light: dark photoperiod, and $35 \%$ salinity. To impose $\mathrm{N}$ deficiency stress, the liquid cultures were discarded, and the microalgae attached to the petri dishes were washed thrice with $\mathrm{f} / 2-\mathrm{N}$ medium and finally re-cultured in $\mathrm{f} / 2-\mathrm{N}$ medium. The other conditions, including temperature, light, and salinity, were not changed.

\subsection{Examination of photosystem II activity}

To monitor the physical status of $A$. coffeaeformis during $\mathrm{N}$ deprivation, the photosynthetic activity as the main representative physical character was measured every $24 \mathrm{~h}$ using Water PAM (WALZ, Germany), while $A$. coffeaeformis cultures in normal $\mathrm{f} / 2$ medium were set as the positive control. The model of slow light curve was used to test the $\mathrm{Y}$ II (Fv/Fm), and Etr. Using different settings, the $A$. coffeaeformis cultures were measured with optimal slow light curve with the following characters. The algae were dark adapted for 10 min to determine the minimal level of fluorescence $\left(F_{0}\right)$ and the maximal fluorescence $\left(F_{m}\right)$ after a saturating flash $\left(0.8 \mathrm{~s} ; 5640 \mu \mathrm{mol} \mathrm{m}^{-2} \mathrm{~s}^{-1}\right)$, and the ratio of variable to maximal 
fluorescence $(\mathrm{Fv} / \mathrm{Fm})$ was calculated. Following a delay of $40 \mathrm{~s}$, the algae were exposed to an actinic illumination of $322 \mathrm{~mol} \mathrm{~m}^{-2} \mathrm{~s}^{-1}$ for $0.8 \mathrm{~s}$ every $20 \mathrm{~s}$ during the $5 \mathrm{~min}$ width.

\subsection{Microscopic observation}

To assess the growth cure of $A$. coffeaeformis, single alga cells were separated using capillaries and monitored using an inverted microscope (200' magnification) every $24 \mathrm{~h}$ to count the cell number. During $\mathrm{N}$ deprivation, the $A$. coffeaeformis cultures were assessed microscopically for community structures, attachment status, and lipid content.

\subsection{Lipid extraction and fatty acid analysis}

A. coffeaeformis cultures were harvested via centrifugation at 5,000' $g$ for $5 \mathrm{~min}$ and freeze-dried (Christ, Germany) for $48 \mathrm{~h}$. Approximately $0.2 \mathrm{~g}$ dry powder was used for total lipid extraction using the ultrasonication and chloroform/methyl alcohol method [37]. Thin layer chromatography analysis of neutral lipids was further performed as described by Kishimoto et al. [38], and a mixture of normal hexane/diethyl ether/acetic acid ( $80 / 20 / 1$ by volume) was used as the mobile phase. Lipid was detected using iodine vapor and quantified using the assay balance. The fatty acid composition was determined using gas chromatography/mass spectrometry as described previously [12].

\subsection{RNA-seq analysis}

After 5 days of $\mathrm{N}$ deprivation, total RNA was extracted from three replicates of $\mathrm{N}$-deprived $A$. coffeaeformis and the control using RNaiso (TAKARA, Dalian, China). The RNA samples were treated with RNase-free DNase I (TAKARA, Dalian, China) and enriched with oligo (dT) magnetic beads. The resulting RNA samples were sequenced by the Novogene company (Beijing, China) using a HiSeq ${ }^{\text {TM }} 2000$ (Illumina) instrument. Owing to the lack of genome sequence, $A$. coffeaeformis RNA was analyzed according to the model diatom Phaeodactylum tricornutum.

\subsection{Differential expression analysis}

Differential expression analysis was performed using the DESeq R package (1.10.1). DESeq provide statistical routines for determining differential expression in digital gene expression data using a model based on the negative binomial distribution. The resulting $P$ values were adjusted using the Benjamini and Hochberg's approach for controlling the false discovery rate. Genes with adjusted P values $<0.05$ found by DESeq were assigned as differentially expressed.

\subsection{Functional enrichment analysis}

Gene Ontology (GO) enrichment analysis of DEGs was implemented using the Gene Ontology Database (http://geneontology.org/) by the GOseq R packages [39]. Kyoto Encyclopedia of Genes and Genomes (KEGG) functional enrichment analysis were performed using KEGG Pathway Database (https://www.genome.jp/kegg/pathway.html) by KOBAS software. 


\subsection{Statistical analysis}

All experiments were performed using biological triplicates to ensure reproducibility. Values are presented as means \pm SD. Statistical analyses were performed using the SPSS statistical package (http://www$01 . i b m . c o m / s o f t w a r e / a n a l y t i c s / s p s s /)$. The paired-samples $t$ test was applied. Differences were considered statistically significant at $P$ values $<0.05$.

\section{Abbreviations}

RNA-seq: RNA-sequencing; TL: total lipid; TAG: triacylglycerol; DW: dry cell weight; PUFAs: polyunsaturated fatty acids; Etr: electron transfer rate; DEGs: differentially expressed genes; TCA: tricarboxylic cycle; CIT: citrate; ICIT: isocitrate; a-KG: a-ketoglutaric acid; SUC: succinate; FUM: fumarate; MAL: malate; OAA: oxaloacetate; GA: glyoxylic acid; PEP: phosphoenolpyruvate.

\section{Declarations}

\section{Acknowledgements and funding information}

The present study was supported by National Natural Science Foundation of China (31972815), Major Basic Research Program for Natural Science Foundation of Shandong Province (ZR2019ZD17, ZR2020ZD23).

\section{Authors' contributions}

Kang Wang: Conceptualization, Software, and Writing- Original draft preparation; Yulin Cui: Validation and Data curation; Chunxiao Meng: Investigation, Methodology, and Funding acquisition; Zhengquan Gao: Writing- Reviewing and Editing, Project administration, and Funding acquisition; Song Qin: Supervision and Funding acquisition.

\section{Availability of data and materials}

The datasets during and/or analyzed during the current study are available from the corresponding author on reasonable request.

Ethics approval and consent to participate. Not applicable.

Consent for publication: Not applicable.

Competing interests. The authors declare that they have no competing interests

\section{References}

1. Kumar M, Sun Y, Rathour R, Pandey A, Thakur IS, Tsang DC. Algae as potential feedstock for the production of biofuels and value-added products: opportunities and challenges. Sci Total Environ. 
2020; 716: 137116.1-137116.17.

2. Jagadevan S, Banerjee A, Banerjee C, Guria C, Shukla P. Recent developments in synthetic biology and metabolic engineering in microalgae towards biofuel production. Biotechnol Biofuels. 2018; 11: 185-205.

3. Shuba ES, Kifle D. Microalgae to biofuels: 'Promising' alternative and renewable energy, review. Renew Sust Energ Rev. 2018; 81: 743-755.

4. Gonalves AL. The use of microalgae and cyanobacteria in the improvement of agricultural practices: a review on their biofertilising, biostimulating and biopesticide roles. Appl Sci. 2021; 11: 871.

5. Milano J, Ong HC, Masjuki HH, Chong WT. Microalgae biofuels as an alternative to fossil fuel for power generation. Renew Sust Energ Rev. 2016; 58: 180-197.

6. Robertson GP, Vitousek PM. Nitrogen in agriculture: balancing the cost of an essential resource. Annu Rev Environ Resour. 2009; 34: 97-125.

7. Schauble EA, Young ED. Mass dependence of equilibrium oxygen isotope fractionation in carbonate, nitrate, oxide, perchlorate, phosphate, silicate, and sulfate minerals. Rev Mineral Geochem. 2021; 86: 137-178.

8. Wu X, Ruan R, Du Z, Liu Y. Current status and prospects of biodiesel production from microalgae. Energies 2012; 5: 2667-2682.

9. Hamed S, Jonathan VW, Per M, Charlotte J. Carotenoids, phenolic compounds and tocopherols contribute to the antioxidative properties of some microalgae species grown on industrial wastewater. Mar Drugs 2015; 13: 7339-7356.

10. Demirbas A. Use of algae as biofuel sources. Energ Convers Manage. 2010; 51: 2738-2749.

11. Jiang $Y$, Yoshida T, Quigg A. Photosynthetic performance, lipid production and biomass composition in response to nitrogen limitation in marine microalgae. Plant Physiol Biochem. 2012; 54: 70-77.

12. Yang C, Zhou J, Liu S, Fan P, Wang W, Xia C. Allelochemical induces growth and photosynthesis inhibition, oxidative damage in marine diatom Phaeodactylum tricornutum. J Exp Mar Biol Ecol. 2013; 444: 16-23.

13. El-Sayed AEKB, Aboulthana WM, El-Feky AM, Ibrahim NE, Seif MM. Bio and phyto-chemical effect of Amphora coffeaeformis extract against hepatic injury induced by paracetamol in rats. Mol Biology Rep. 2018; 45: 2007-2023.

14. Taipale S, Peltomaa E, Salmi P. Variation in $\omega-3$ and $\omega-6$ polyunsaturated fatty acids produced by different phytoplankton taxa at early and late growth phase. Biomolecules 2020; 10: 559.

15. Chtourou H, Dahmen I, Jebali A, Karray F, Hassairi I, Abdelkafi S, Ayadi H, Sayadi S, Dhouib A. Characterization of Amphora sp. a newly isolated diatom wild strain, potentially usable for biodiesel production. Bioproc Biosyst Eng. 2015; 38: 1381-1392.

16. Indrayani I, Moheimani NR, Boer KD, Bahri PA, Borowitzka MA. Temperature and salinity effects on growth and fatty acid composition of a halophilic diatom, Amphora sp. MUR258 (Bacillariophyceae). J Appl Phycol. 2020; 32: 977-987. 
17. Gruber-Brunhumer M, Nussbaumer M, Jerney J, Ludwig I, Zohar E, Lang I, Bochmann G, Schagerl M, Obbard JP, Fuchs W. Two-stage cultivation of N-rich and N-deprived Acutodesmus obliquus biomass: influence of cultivation and dewatering methods on microalgal biomass used in anaerobic digestion. Algal Res. 2016; 17: 105-112.

18. Berges JA, Falkowski PG. Physiological stress and cell death in marine phytoplankton: induction of proteases in response to nitrogen or light limitation. Limnol Oceanogr. 1998; 43: 129-135.

19. Converti A, Casazza AA, Ortiz EY, Perego P, Borghi MD. Effect of temperature and nitrogen concentration on the growth and lipid content of Nannochloropsis oculata and Chlorella vulgaris for biodiesel production. Chemi Eng Process 2009; 48: 1146-1151.

20. Peltier G, Schmidt G. Chlororespiration: an adaptation to nitrogen deficiency in Chlamydomonas reinhardtii. Proc Natl Acad Sci. 1991; 88: 4791-4795.

21. Hockin NL, Mock T, Mulholland F, Kopriva S, Malin G. The response of diatom central carbon metabolism to nitrogen starvation is different from that of green algae and higher plants. Plant Physiol. 2012; 158: 299-312.

22. Roberts K, Granum E, Leegood R, Raven J. Carbon acquisition by diatoms. Photosyn Res. 2007; 93 : 79-88.

23. Song P, Zhang L, Li Q. Response of photosynthetic apparatus of Isochrysis galbana to different nitrogen concentrations. Bioresour Technol Rep. 2018; 4: 74-79.

24. Wase N, Tu B, Rasineni G, Grove R, Dirusso CC. Remodeling of Chlamydomonas metabolism using synthetic inducers results in lipid storage during growth. Plant Physiol. 2019; 181: 1029-1049.

25. Turpin DH, Elrifi IR, Birch DG, Weger HG, Holmes JJ. Interactions between photosynthesis, respiration, and nitrogen assimilation in microalgae. Can J Bot. 1988; 66: 2083-2097.

26. Campbell D, Hurry V, Clarke A, Gustafsson P, Oquist G. Chlorophyll fluorescence analysis of Cyanobacterial photosynthesis and acclimation. Microbiol. Mol Biol Rev. 1998; 62: 667-683.

27. Arrivault S, Moraes TA, Obata T, Medeiros DB, Fernie AR, Boulouis A, Ludwig M, Lunn JE, Borghi GL, Schlereth A, Guenther M, Stitt M. Metabolite profiles reveal inter-specific variation in operation of the Calvin-Benson cycle in both C4 and C3 plants. J Exp Bot. 2019; 70(6).

28. Karolina H, Ray JN, Ayyampalayam S, Moledina N, Borland A, Harding SA, Tsai CJ, Jim LM. Shared expression of crassulacean acid metabolism (cam) genes predates the origin of cam in the genus yucca. J Exp Bot. 2019; 70: 6597-6609.

29. Kosourov SN, Nagy V, Shevela D, Jokel M, Allahverdiyeva Y. Water oxidation by photosystem ii is the primary source of electrons for sustained h2 photoproduction in nutrient-replete green algae. Proc Natl Acad Sci. 2020; 117: 29629-29636.

30. Hopkinson B, Dupont C, Allen A, Morel F. Efficiency of the CO2-concentrating mechanism of diatoms. Proc Natl Acad Sci USA. 2011; 108: 3830-3837.

31. Wang H, Liu C, Ma P, Lu C, Li K, Wang W. Functional characterization of cytosolic pyruvate phosphate dikinase gene (MecyPPDK) and promoter (MecyPPDKP) of cassava in response to abiotic stress in transgenic Tobacco. Crop Sci. 2018; 58: 2002-2009. 
32. Chang TS, Liu CW, Lin YL, Li CY, Wang AZ, Chien MW, Wang CS, Lai CC. Mapping and comparative proteomic analysis of the starch biosynthetic pathway in rice by 2D PAGE/MS. Plant Mol Biol. 2017; 95: 333-343.

33. Jeon S, Koh HG, Cho JM, Kang NK, Chang YK. Enhancement of lipid production in Nannochloropsis salina by overexpression of endogenous NADP-dependent malic enzyme. Algal Res. 2021; 54 : 102218.

34. Xue J, Wang L, Zhang L, Balamurugan S, Li DW, Zeng H, Yang WD, Liu JS, Li HY. The pivotal role of malic enzyme in enhancing oil accumulation in green microalga Chlorella pyrenoidosa. Microb Cell Fact. 2016; 15: 1-9.

35. Imamura S, Kawase Y, Kobayashi I, Sone T, Era A, Miyagishima S, Shimojima M, Ohta H, Tanaka K. Target of rapamycin (TOR) plays a critical role in triacylglycerol accumulation in microalgae. Plant Mol Biol. 2015; 89: 309-318.

36. Fukuda S, Hirasawa E, Takemura T, Takahashi S, Chokshi K, Pancha I, Tanaka K, Imamura S. Accelerated triacylglycerol production without growth inhibition by overexpression of a glycerol-3phosphate acyltransferase in the unicellular red alga Cyanidioschyzon merolae. Sci Rep. 2018; 8: 12410.

37. Zhang X, Yan S, Tyagi RD, Drogui P, Surampalli RY. Ultrasonication assisted lipid extraction from oleaginous microorganisms. Bioresource Technol. 2014; 158: 253-261.

38. Kishimoto K, Urade R, Ogawa T, Moriyama T. Nondestructive quantification of neutral lipids by thinlayer chromatography and laser-fluorescent scanning: suitable methods for "lipidome" analysis. Biochem Bioph Res Co. 2001; 281: 657-662.

39. Young MD, Wakefield MJ, Smyth GK, Oshlack A. Gene ontology analysis for RNA-seq: accounting for selection bias. Genome Biolo. 2010; 11: R14.

\section{Figures}




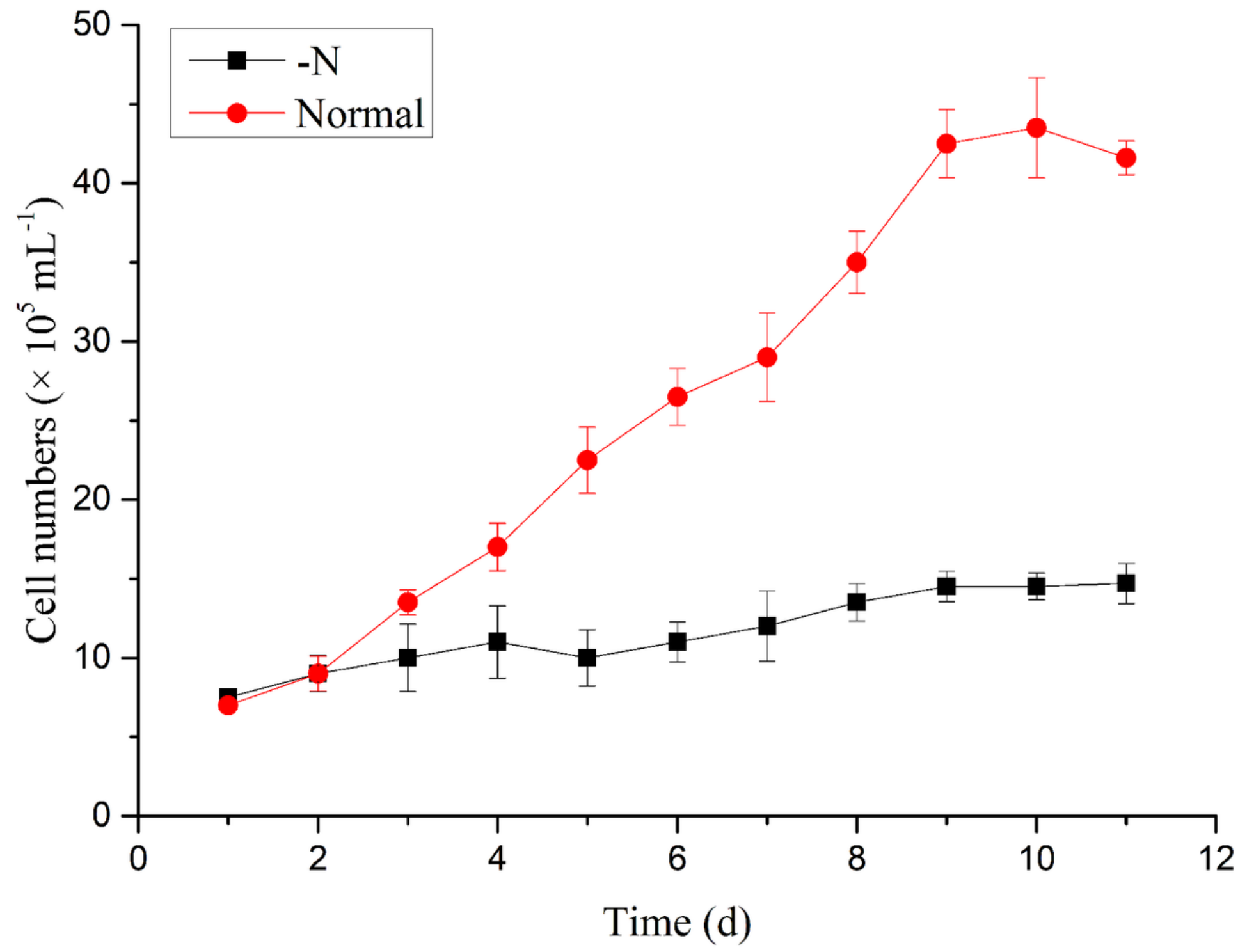

Figure 1

Growth of A. coffeaeformis under N-deprived and normal conditions at $30^{\circ} \mathrm{C}$ with $12 \mathrm{~h} / 12 \mathrm{~h}$ light cycle and $100-110 \mu \mathrm{mol} / \mathrm{m} 2 \mathrm{~s}-1$ light intensity. $-\mathrm{N}$, cells were cultured at $\mathrm{N}$-deprived f/2 medium; Normal, cells were cultured at normal f/2 medium. Each value represents mean $\pm S D(n=3)$ 


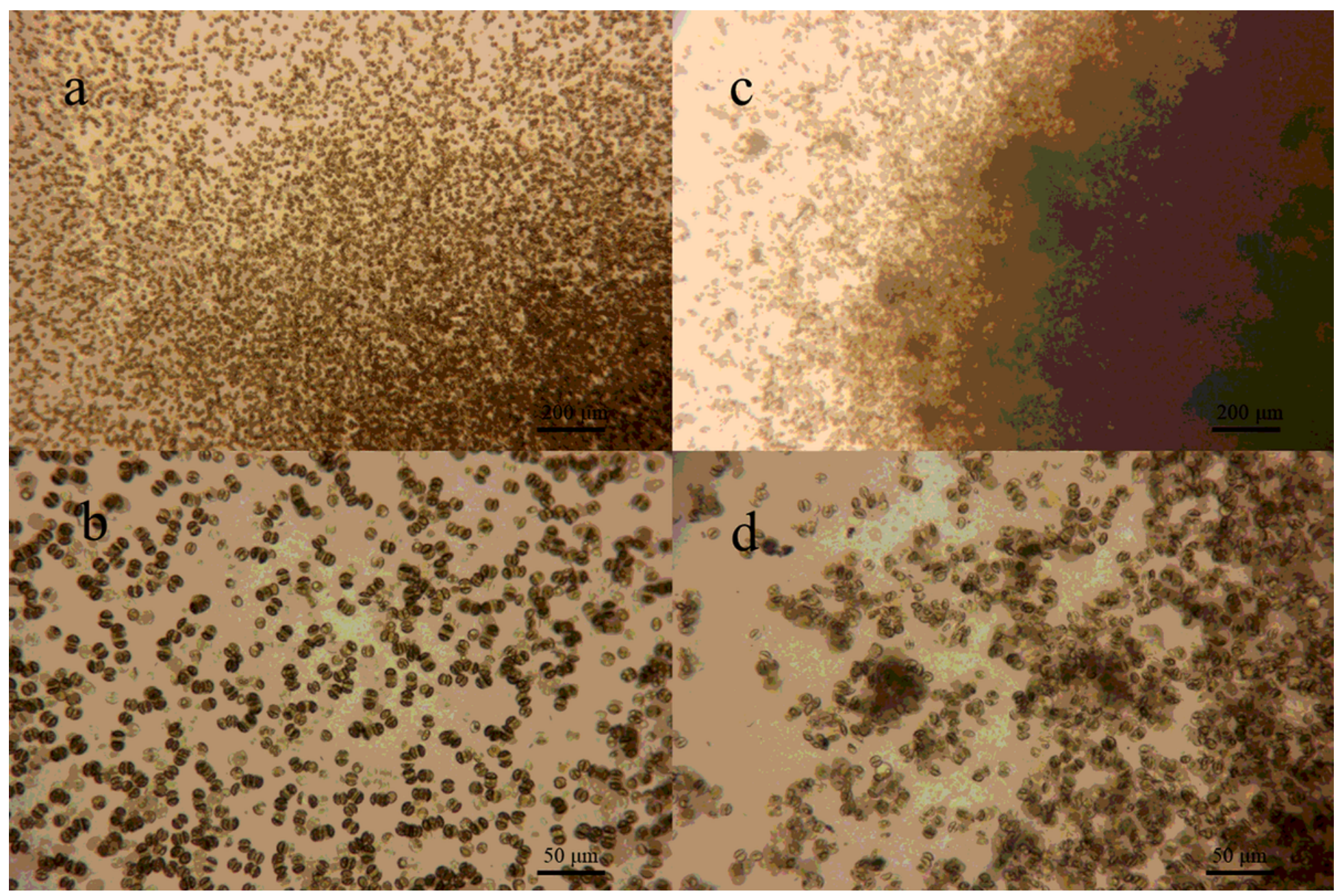

Figure 2

The attachment status of A. coffeaeformis cultured on normal medium or under N-deprived medium. a, Culture with normal medium, 4×10 magnified; b, Culture with normal medium, 10×10 magnified; c, Culture inducted by $\mathrm{N}$ deprived $\mathrm{f} / 2$ medium for 7 days, $4 \times 10$ magnified; $d$, Culture inducted by $\mathrm{N}$ deprived $\mathrm{f} / 2$ medium for 7 days, $10 \times 10$ magnified. 

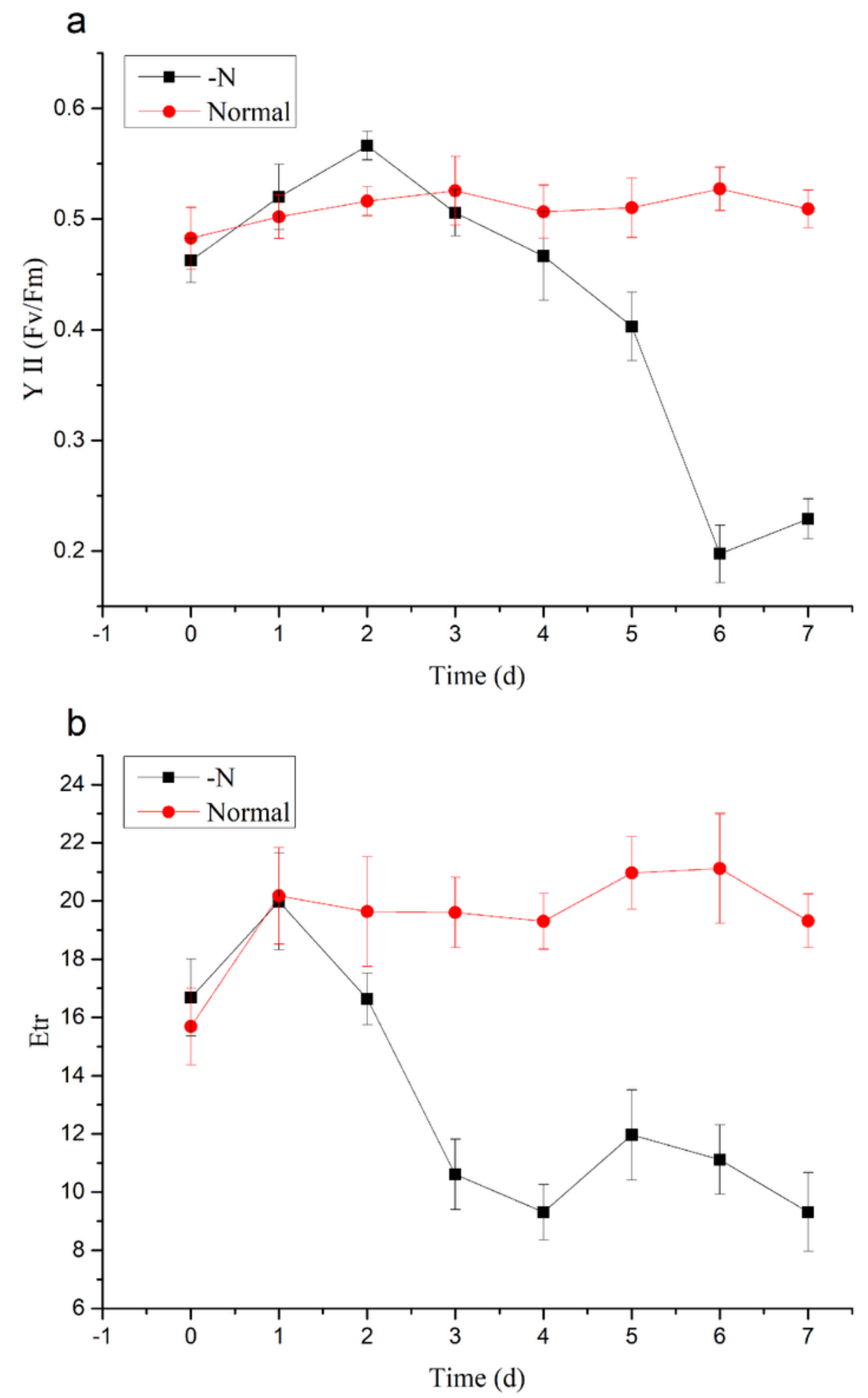

\section{Figure 3}

Photosynthetic efficiency of A. coffeaeformis under normal and N-deprived (-N) conditions. a, The maximum PSIl photochemical efficiency (Fv/Fm); b, electron transfer rate (Etr). Each value represents mean $\pm S D(n=3)$. 


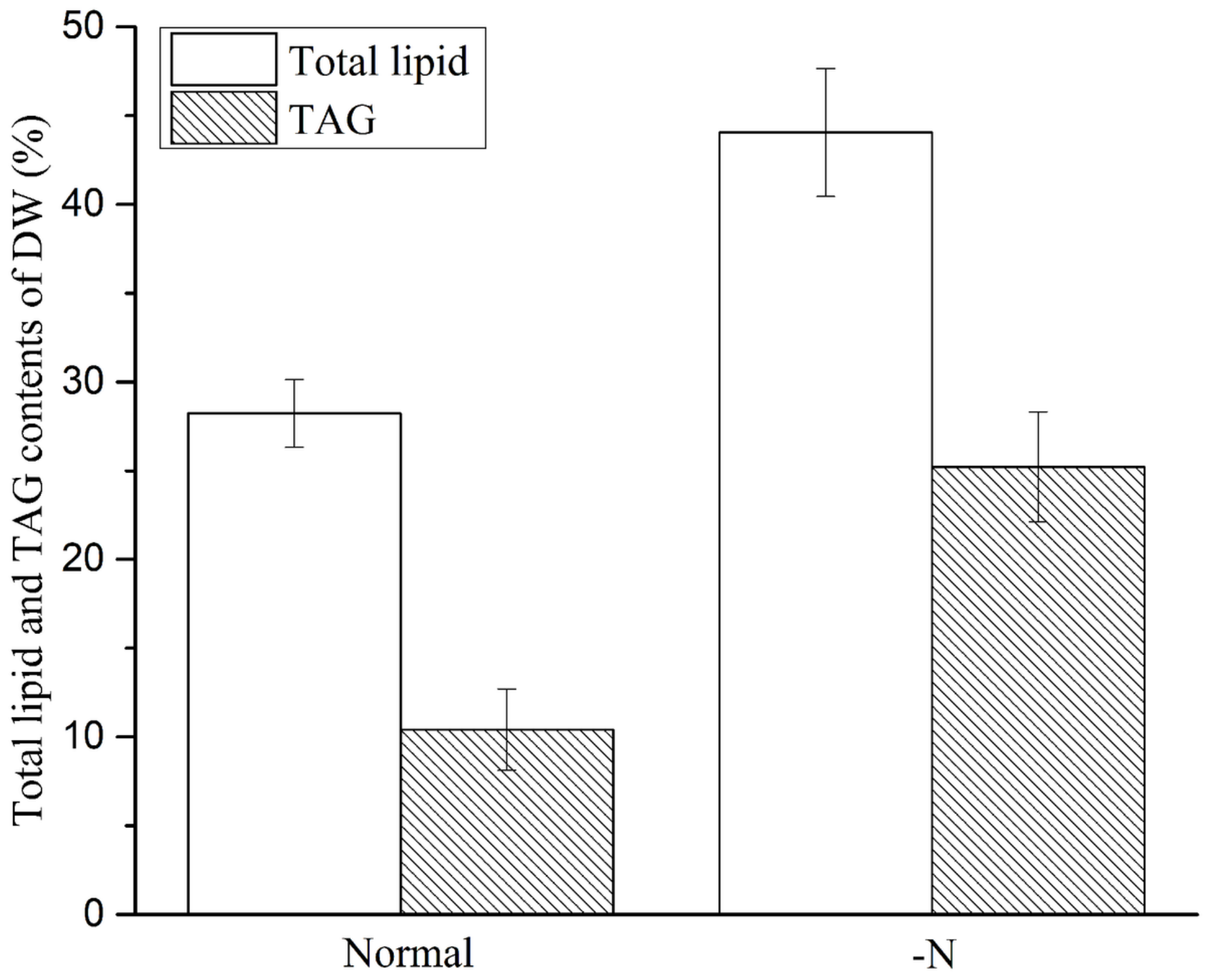

Figure 4

Total lipid and TAG contents under normal and N-deprived conditions. Each value represents mean \pm SD $(n=3)$. 
Environmental adaptation

Overview

Nucleotide metabolism

Metabolism of terpenoids and polyketides Metabolism of other amino acids Metabolism of cofactors and vitamins

Lipid metabolism

Glycan biosynthesis and metabolism

Energy metabolism

Carbohydrate metabolism

Biosynthesis of other secondary metabolites

Amino acid metabolism Translation Transcription

Replication and repair

Folding, sorting and degradation

Signal transduction

Membrane transport

Transport and catabolism

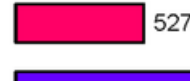

527

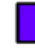
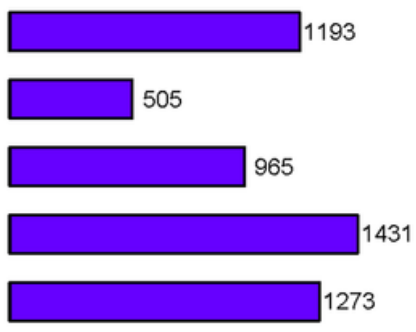

131
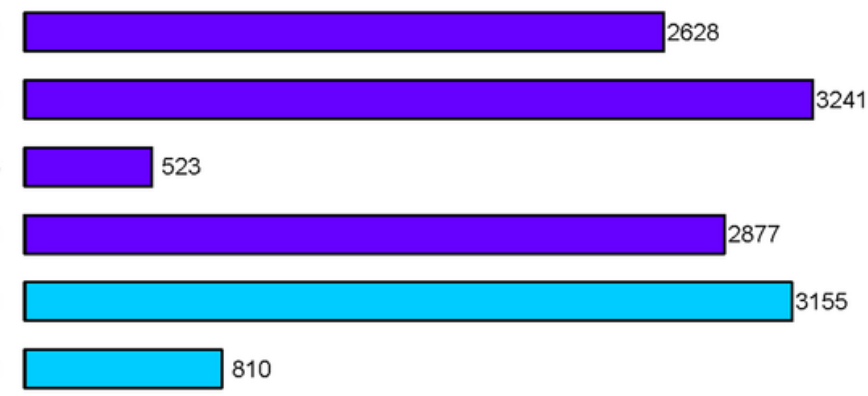

$\square 350$
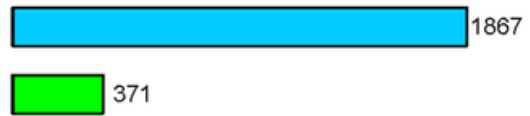

$\square 210$

371

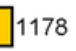

\begin{tabular}{|c|c|c|c|c|}
\hline & $T$ & $T$ & $T$ & 7 \\
\hline 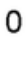 & 5 & $\begin{array}{c}10 \\
\text { Percen }\end{array}$ & 15 & 20 \\
\hline
\end{tabular}

\section{Figure 5}

KEGG classification of the assembled transcripts. A, cellular processes; $B$, environmental information processing; $C$, genetic information processing; $D$, general metabolism; E, organismal systems. 
a

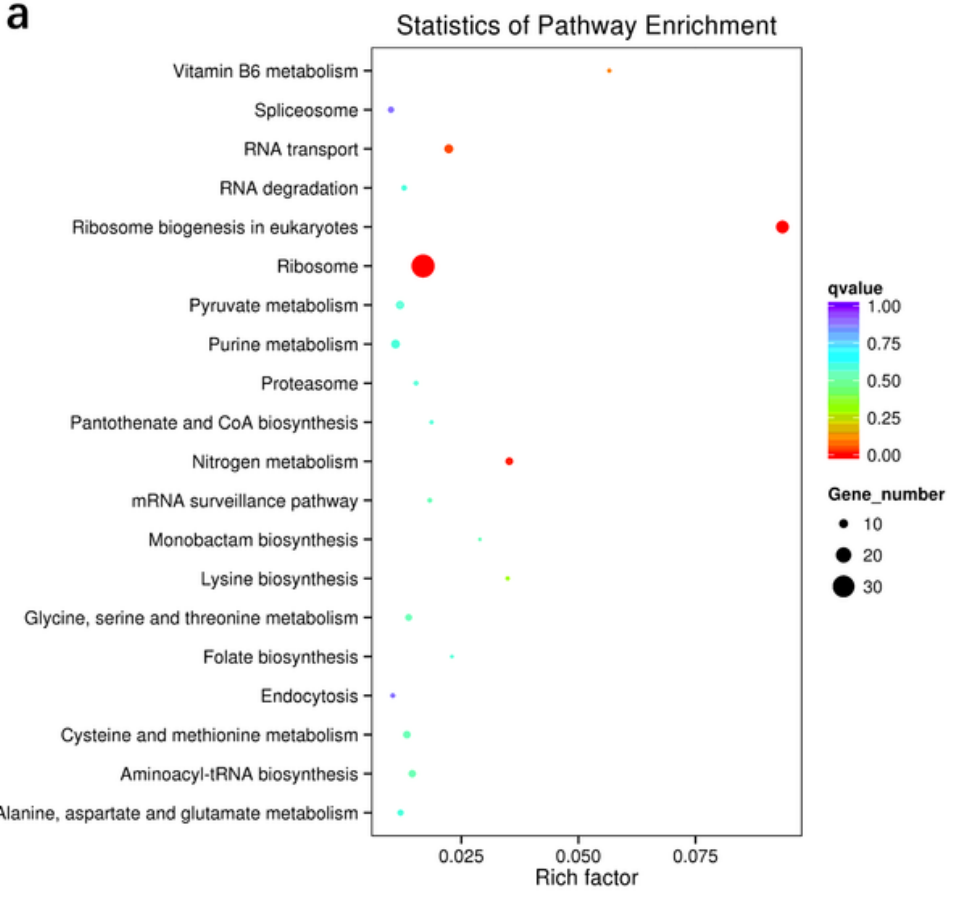

b

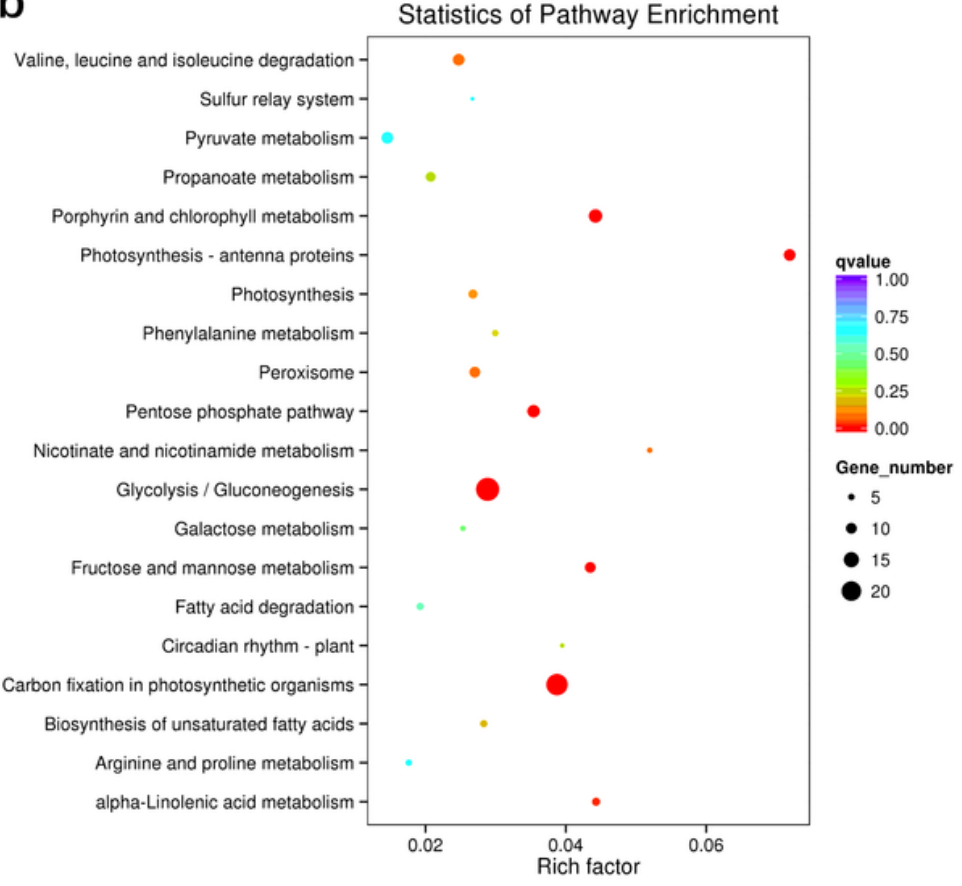

Figure 6

KEGG enrichment analyses of DEGs of A. coffeaeformis. a, KEGG pathway analysis of up-regulated DEGs; b, KEGG pathway analysis of down-regulated DEGs. 


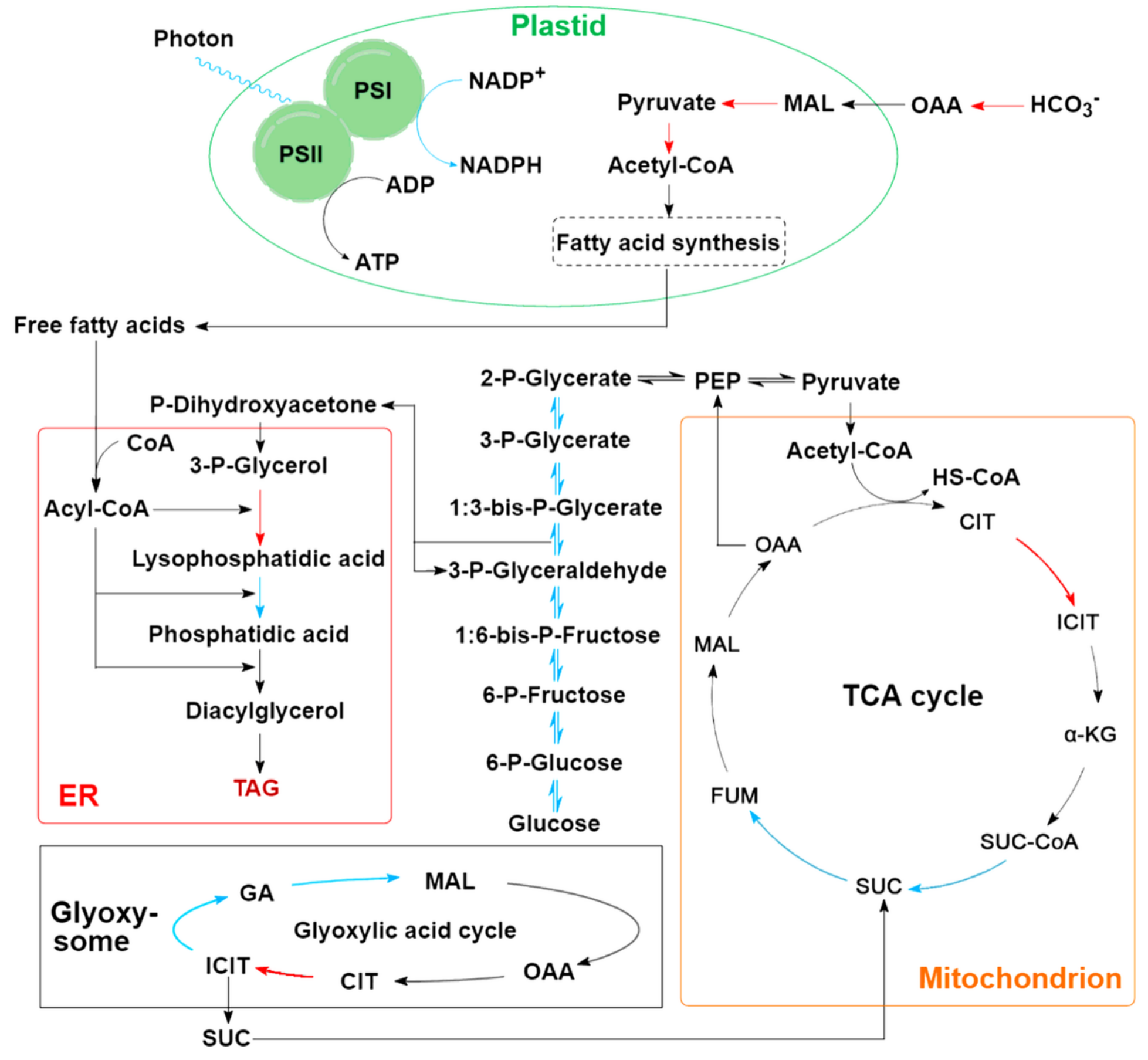

Figure 7

Proposed general transcriptional changes of $\mathrm{A}$. coffeaeformis under $\mathrm{N}$ deprivation. Schematic diagram showing the putative localization of central metabolic pathways of A. coffeaeformis.

\section{Supplementary Files}

This is a list of supplementary files associated with this preprint. Click to download. 
- Additionalfile1.docx

- Additionalfile2.docx

Page 25/25 